\title{
PAPR Reduction Using Huffman and Arithmetic Coding Techniques in F-OFDM System
}

\author{
Azlina Idris', Nur Atiqah Md Deros², Idris Taib ${ }^{3}$, Murizah Kassim4, Mohd Danial Rozaini ${ }^{5}$, \\ Darmawaty Mohd Ali ${ }^{6}$ \\ 1,2,4,5,6 Faculty of Electrical Engineering, Universiti Teknologi MARA (UiTM), Wireless Communication Technology \\ (WiCOT), 40450 Shah Alam, Selangor, Malaysia \\ ${ }^{3}$ Infrastructure University Kuala Lumpur, 43000 Kajang, Selangor, Malaysia
}

\begin{tabular}{l} 
Article Info \\
\hline Article history: \\
Received Jan 31, 2018 \\
Revised Mar 31, 2018 \\
Accepted Apr 14, 2018 \\
\hline Keywords: \\
Arithmetic coding and huffman \\
coding \\
Block coding technique \\
Filtered Orthogonal Frequency \\
Division Multiplexing (F- \\
OFDM) \\
Orthogonal Frequency Division \\
Multiplexing (OFDM) \\
\hline
\end{tabular}

\begin{abstract}
Filtered orthogonal frequency division multiplexing (F-OFDM) was introduced to overcome the high side lobes in the OFDM system. Filtering is implemented in the system to reduce the out-of-band emission (OOBE) for the spectrum utilization and to meet the diversified expectation of the upcoming 5G networks. The main drawback in the system is the high peak to average ratio (PAPR). This paper investigates the method used in reducing the PAPR in the F-OFDM system. The proposed method using the block coding technique to overcome the problem of high PAPR are the Arithmetic coding and Huffman coding. This research evaluates the performance of FOFDM system based on the PAPR values. From the simulation results, the PAPR reduction of the Arithmetic coding is $8.9 \%$ lower, while the Huffman Coding is $6.7 \%$ lower in the F-OFDM system. The results prove that the Arithmetic Coding will out-perform the Huffman coding in the F-OFDM system.
\end{abstract}

Copyright $@ 2018$ Institute of Advanced Engineering and Science. All rights reserved.

\section{Corresponding Author:}

Azlina Idris,

Faculty of Electrical Engineering,

Universiti Teknologi MARA (UiTM),

Wireless Communication Technology (WiCOT),

40450 Shah Alam, Selangor, Malaysia.

Email: azlina831@salam.uitm.edu.my

\section{INTRODUCTION}

The Orthogonal frequency division multiplexing (OFDM) system has been widely used in many latest wireless and telecommunications standards in the area of the wireless communication and 4G networks due to the high demand of the high data rate communications [1]. This wideband digital communication has been used in both wired and unwired applications as the signal modulation that divides the high data rates modulating stream [2]. Besides that, the OFDM is good for point to point application that offers a minimum complexity and good bandwidth efficiency [3]. At present, research and development for the fifth generation cellular communication (5G) network are full in action to overcome the problem that arises from the conventional OFDM system. The futures $5 \mathrm{G}$ are expected to be more flexible on the usage of limited spectrum to reduce the guard bands and improve the efficiency [4].

The conventional OFDM are also being recognized to suffer from the high out-of-band (OOB) leakage that disables the coexistence of multiple services in the system [5]. To prevail over the issue, the Filtered OFDM (F-OFDM) is being introduced to meet the expectations of the 5G networks. The lower the out-of-band (OOB) power, the higher the spectral efficiency in the system [4], [6]. In addition, the F-OFDM could help to reduce spectrum efficiency over the OFDM that has capability to support the multiple 
asynchronous sub-band transmission [7]. The aim of the F-OFDM system is to get high spectrum efficiency for ultra reliability and low latency communication with larger subcarrier spacing [8]. The filtering will help to suppress the OOB leakage. The Finite Impulse Response (FIR) digital filter based on the windowing functional method was used in the F-OFDM to divides the bandwidth in the system into a number of sub-bands [9].

The main drawback of using the F-OFDM system is the high PAPR when the signal passes through non-linear high power amplifier (HPA) at the receiver [10]. The non- linear effect will be more sensitive when the F-OFDM has large dynamic signal and large number of subcarrier with the PAPR [11]. While there are various methods may be used overcome high PAPR, this research uses the Block Coding technique to solve the problem [12]. The Arithmetic coding and Huffman coding was compared as the Block coding technique using the lossless data compression in the encoding process. The data that being encoded is depend on the statically frequency probability. Thus, the expected results shall demonstrate that Arithmetic scheme will outperform Huffman codes with same number bits per symbols in the F-OFDM system.

Even though many researchs have been conducted to overcome the PAPR reduction using block coding technique for OFDM system, there are still no research has been made using the F-OFDM system. By using the F-OFDM the secondary user are allow to share the portions of the unoccupied spectrum to get greater spectral efficiency utilization [13]. This paper evaluate the performance of PAPR for the F-OFDM system using the block coding techniques which are the Arithmetic coding and Huffman coding.

In section 2 the system model is presented while section 3 provides the software development and the mathematical formulations for the F-OFDM system. Section 4 deliberates the simulation and analyse the result. The findings are then conculded in section 5.

\section{SYSTEM MODEL}

\subsection{Filtered-orthogonal Frequency Division Multiplexing (F-OFDM)}

Figure 1 above show the block diagram of the F-OFDM transceiver system. Filtered OFDM (FOFDM) is used to full fill the expectation of the upcoming $5 \mathrm{G}$ cellular network and being applied to a subband consisting of a group of subcarriers. The system bandwidth is separated into several sub-bands to a certain width and each sub-band is filtered separately [13]. The sum of the filtered sub-band is transmitted depending on the allocated frequency resources to improve the spectrum utilization [7], [13], [14]. The 5G system should be able to offer better services to different channel characteristics [9]. Besides that, the FOFDM should also be able to reduce the out-of-band (OOB) leakage that tolerate to the time frequency misalignment and able to overcome the drawback of the system [7], [13].

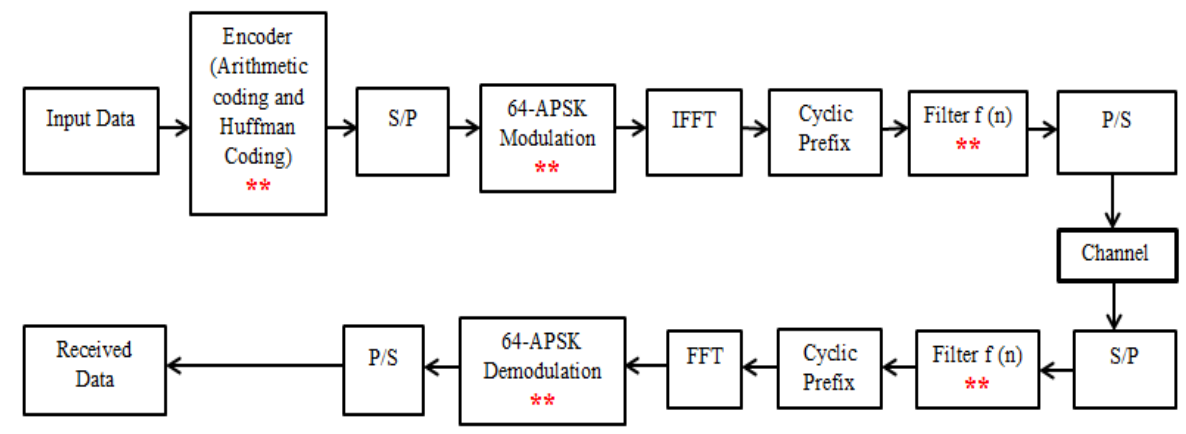

Figure 1. Block diagram of OFDM transceiver system [9]

F-OFDM undergoes similar process as the conventional OFDM. The only process that differentiates both of the system is the filter processes that being applied after the cyclic prefix process. In the F-OFDM, the sub-band based filtering is applied to suppress the inter-subband interference to get lower OOBE with negligible performance loss [15]. The choices of the filter use are depending on the filter that able to minimize the OOB radiation with a constant pass band [13], [16]. The filter used must keep longer filter impulse response length compared to the cyclic prefix length. As the filter tailed exceeds the cyclic prefix length, there will be a certain inter-symbol interference (ISI) even for a flat channel [17].

In this paper, the Finite Impulse Response (FIR) filter is being chosen because of the fast, convenient and robust sub-optimal [9]. FIR filter is using the windowing technique and has a finite duration because it settles to zero in a finite time [9]. The ISI can be minimized without loosing the efficiency [8]. The 
F-OFDM system may result in a lower PAPR reduction and a better performance of the BER since it consider as the biggest hurdle of the OFDM system which might increase the efficiency of the system.

\subsection{Finite Impulse Response (FIR) Filter}

The filter impulse response (FIR) depends on the windowing of the filter which is known as nonrecursive digital filters since it does not have any feedback [18]. In designing the FIR filter, the transfer function of the FIR filter will approach the ideal filter when the order of the filter increases [19]. This will increase the complexity and the amount of time needed to process the input data that being filter in the F-OFDM system.

The result of the frequency response can monotone the function within a certain frequency range and the waveform of the frequency response usually depend on the method that being implement in the filtering [9]. This research implements the windowing technique in the filtering scheme. The characteristics of the transfer function depend on the window function used in the F-OFDM system. There are various type of windowing that can be applied in the F-OFDM system such as the rectangular window, hanning window and hamming window. The equation of the window type is shown in Table 1.

Table 1. Windowing Type Equation [19]

\begin{tabular}{cc}
\hline Window Type & Equation \\
\hline Rectangular & $w(n)=1$ \\
Hanning & $w(n)=0.5-0.5 \cos \left(\frac{2 \pi n}{M}\right)$ \\
Hamming & $w(n)=0.54-0.46 \cos \left(\frac{2 \pi n}{M}\right)$ \\
\hline
\end{tabular}

This window method develop the causal linear phase FIR filter by multiplying an ideal filter transfer function, $h_{d}[n]$ with the windowing function, w[n] as in [19].

$$
h[n]=h_{d}[n] w[n]
$$

Windowing is a way to reduce the interference in the F-OFDM system. The windowing process is used to suppress the discontinuity of the signal by removing the mean value from the signal [20].

\subsection{Arithmetic Coding Technique}

Arithmetic coding is a technique used for data compression in the form of entropy coding used for lossless data compression [20]. The length of the arithmetic coding depends on the statistical frequency that makes the arithmetic coding to have the probability equalization capabilities and produce lower PAPR value than other encoder technique [21]. This encoding process is an incremental process as each character is encoded and a few bits will be added to build up the data over time as the algorithm proceeds. As the compression process begin the coding range will has 0 and 1 to represent low and high ends of the input data [11]. Then, a cumulative probability interval $\left(Q_{x}, Q_{x+1}\right)$ is located to every possible input symbol. Every time the symbol is being encoded, the coding range will be narrowed to a portion allocated to the symbol according to its cumulative probability interval [12]. The arithmetic encoder is being used to calculate the new lower end and higher end of the coding range using the equation [11];

$$
\begin{aligned}
& \text { high }_{\text {new }}=\text { low }_{\text {old }}+\text { range }_{\text {old }} \times Q_{x+1} \\
& \text { low }_{\text {new }}=\text { low }_{\text {old }}+\text { range }_{\text {old }} \times Q_{x}
\end{aligned}
$$

Where, range $_{\text {old }}=$ high $_{\text {old }}-$ low $_{\text {old }}$ or simply the new high end of the coding range the new coding range can be obtained [11];

$$
\text { range }_{\text {new }}=\text { range }_{\text {old }}-P_{x}
$$

\subsection{Huffman Coding Technique}

Huffman coding is a data compression technique that varies according to the length of the encoded symbol in portion to its content information [22]. It is a lossless data compression algorithm and the variable length codes assigned to the input are a prefix codes. The coding technique uses lower number of bits to encode the data that occurs more frequently. Binary string is used to represent the compress string in the system and to reduce the overall number of bits used to encode the string. The Huffman coding is then used to find the minimum length of bit string which can be used to encode the string of symbols [23], [24]. In 
other words, Huffman coding uses the table of frequency occurrence to derive the input from processing a large number of symbols [23]. Huffman coding technique is called minimum redundancy codes. This Huffman coding causes the probability density to fluctuate around [12]. Figure 2 show the example of tree diagram for Huffman Compression Technique.

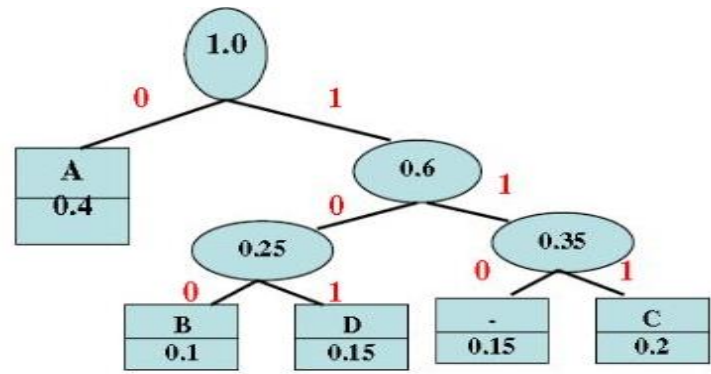

Figure 2. An example of Huffman compression technique tree diagram

\section{METHODOLOGY}

\subsection{Flowchart}

The Figure 3 above illustrates the system simulation development process throughout the completion of the project. All simulations were done using MATLAB software. The first step is to initialize the system parameters as presented in Table 2 below. Next, apply the Arithmetic coding and Huffman coding technique in the encoder using random input sources for the encoding process. Then, implement F-OFDM system according to the system block diagram as shown in Figure 1. As for the F-OFDM systems, the filter was tested using the windowing technique such as the rectangular window, hanning window and hamming window. In order to evaluate the system performance, PAPR were plotted. The simulation results obtained is then being compared with the theoretical framework. Analysis from the simulations is done in order to examine the best system that meets the research objectives.

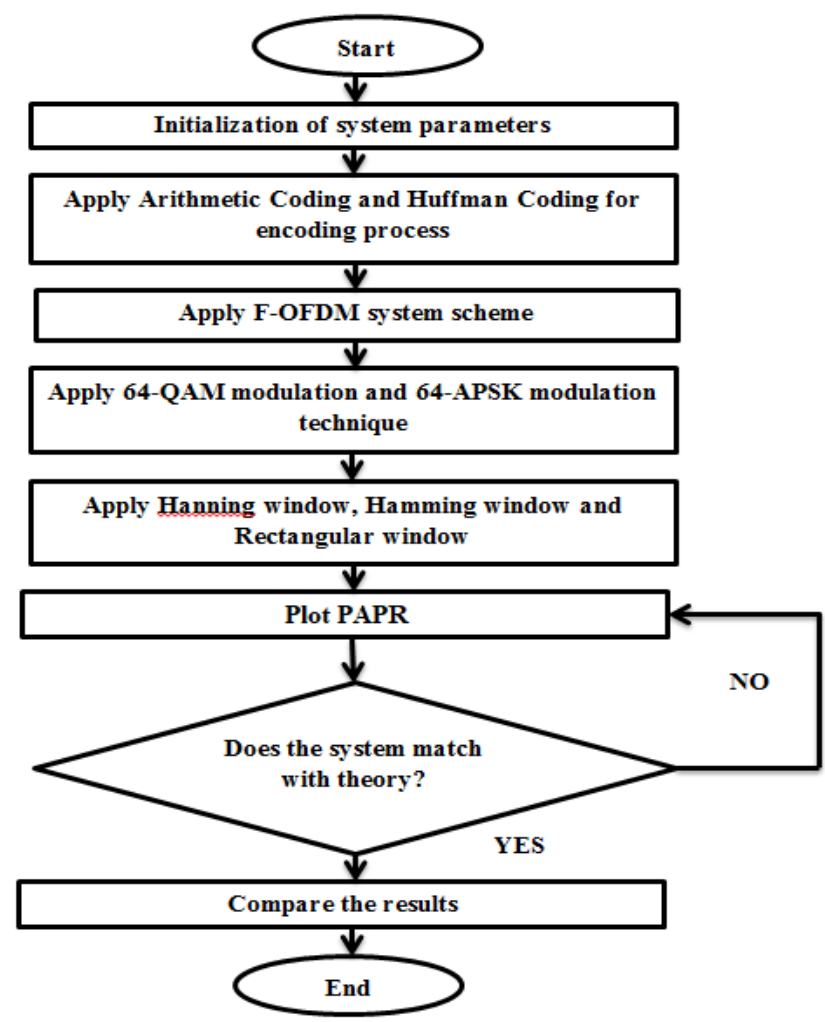

Figure 3. Software development flowchart 


\section{RESULTS AND DISCUSSION}

The simulation results of BER and PAPR for F-OFDM system are presented in this section as shown below. Table 2 shows all the parameters used for simulations of F-OFDM. The CCDF will be used to monitor the F-OFDM system regarding the reduction of the PAPR.

Table 2. System Parameters [8]

\begin{tabular}{lc}
\hline \multicolumn{1}{c}{ Parameter } & Specification \\
\hline Channel model & Rayleigh \\
Bandwidth & $10 \mathrm{MHz}$ \\
Modulation scheme & $64 \mathrm{APSK}$ \\
FFT size & 4096 \\
Cyclic prefix modes & $(1 / 4)^{* 4096}$ \\
Filter Order & 256 \\
\hline
\end{tabular}

\subsection{PAPR Performance Using F-OFDM Without Block Coding Technique}

Figure 4 illustrate the plot of distribution (CCDF) for PAPR F-OFDM system to compare various type of modulation that is the APSK and QAM. Table 3 shows PAPR readings from the simulation. PAPR value of F-OFDM using APSK modulation at $10^{-1}$ is $32.7 \%$ better than the threshold value. For F-OFDM using QAM modulation technique, the PAPR is $28.8 \%$ better meanwhile it is $4.8 \%$ lower PAPR in OFDM system with APSK modulation. Since F-OFDM with APSK modulation technique shows the highest percentage of improvement in PAPR compared to the other systems, it is proved that this scheme is capable to increase power efficiency in the system.

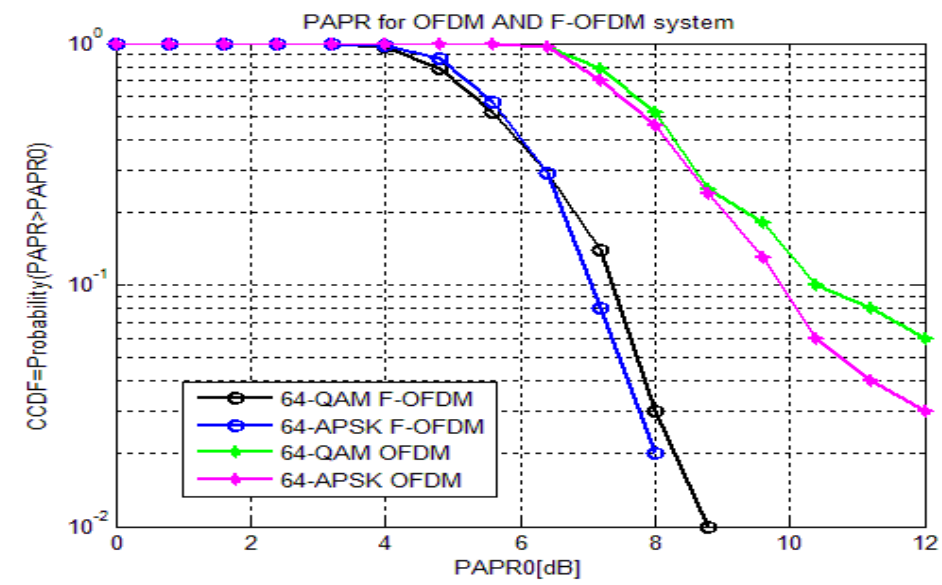

Figure 4. PAPR of F-OFDM system using different modulation technique.

Table 3. PAPR values for F-OFDM System

\begin{tabular}{ccccc}
\hline & OFDM (64-QAM) & F-OFDM (64-QAM) & OFDM (64-APSK) & F-OFDM (64-APSK) \\
\hline PAPR (dB) & 10.4 & 7.4 & 9.9 & 7.0 \\
\hline
\end{tabular}

From the simulation graph in Figure 4, it is clearly demonstrated that the 64 APSK F-OFDM overcome the performance in PAPR in the system. This shows that the APSK modulation is a promising solution for power efficiency because of the high spectrum efficiency and has smaller fluctuation compare to QAM [25]. Besides that, the non linear will help to reduce the inter-symbol interference (ISI). The APSK will show lower power loss and allow reducing of the output back-off respect to the QAM modulation. The APSK modulation can be used with the grey coding to improve PAPR performance. The modulation also reduces the out-of band radiation especially when having strong non linear distortion due to high amplitude. The performance improvement is achieved with no further complexity in the transmitter and receiver. As for the QAM, it suffers from high envelope fluctuation that resulting in a non linear distortion of the transmitting signal due to high power amplifier (HPA) [25]. This makes the QAM modulation less efficient and and makes them less attractive to be implementing for any application [25]. 


\subsection{PAPR Performance Using F-OFDM with Coding Technique}

Figure 5 illustrate the plot of distribution (CCDF) for PAPR F-OFDM system with different type of modulation techniques that are the Arithmetic coding and Huffman coding. Table 4 shows PAPR readings from the simulation. PAPR value of F-OFDM using Arithmetic coding at $10^{-1}$ is $8.9 \%$ higher than the threshold value. For F-OFDM using Huffman coding, the PAPR is $6.7 \%$ better meanwhile it is $1.1 \%$ lower PAPR in OFDM using Arithmetic coding. Since F-OFDM system using Arithmetic coding shows the highest percentage of improvement in PAPR compared to the other systems, it is proved that this scheme is capable to increase power efficiency in the system.

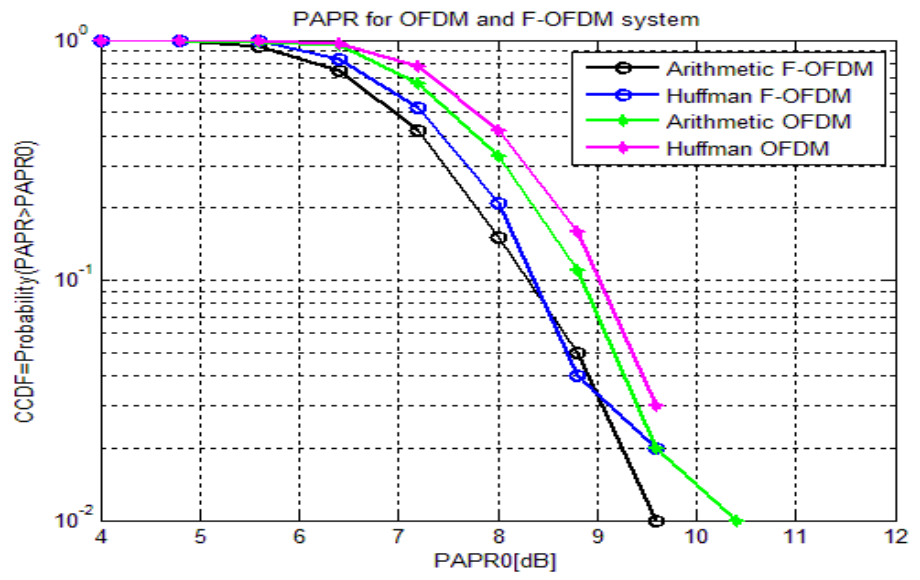

Figure 5. PAPR for SCFDMA system with different decoding techniques

Table 4. PAPR values for F-OFDM System

\begin{tabular}{ccccc}
\hline & OFDM (Huffman) & OFDM (Arithmetic) & F-OFDM (Huffman) & F-OFDM (Arithmetic) \\
\hline PAPR (dB) & 9.0 & 8.9 & 8.4 & 8.2 \\
\hline
\end{tabular}

Based on the illustrated result in the Figure 5, it can be concluded that the Arithmetic in F-OFDM system show the highest improvement for PAPR reduction because the Arithmetic coding have advantages over the lookup table for encoding and decoding process [12]. The probability distribution will be greatly equalized using arithmetic coding compared to the Huffman coding. This Huffman coding tends to cause the probability to fluctuate around. And need an exhaustive starch through the code work that make the storing of the lookup table to be larger than Arithmetic coding [23]. From the simulation results, the Arithmetic Coding has been chooses to be the Probability Distribution Equalization (PDE) due to the high reduction of the PAPR in the system [12].

\section{CONCLUSION}

In this research, the performance of F-OFDM system has been compared with the OFDM system. The F-OFDM is an enabler to get a flexible waveform and implement to meet the requirement of the latest 5G networks. The key feature of an efficient system for F-OFDM is to get higher PAPR reduction. It was found that by applying the hanning window technique and 64-APSK modulation technique in the F-OFDM system produce better results as it can reduce the PAPR. The obtained result has demonstrated that the Arithmetic coding outperform the Huffman coding in PAPR reduction. From the above analysis, the PAPR in the F-OFDM system has been reduced by $32.0 \%$ and $30.6 \%$ in the Arithmetic coding and the Huffman coding respectively. Therefore, the Arithmetic coding is highly recommended as the PDE.

\section{ACKNOWLEDGEMENT}

This works was partly supported by the Faculty of Electrical Engineering, Universiti Teknologi Mara, Shah Alam, Selangor, Malaysia, and Ministry of Higher Education, Malaysia (research grant FRGS/1/2015/TK04/UITM/02/25). 


\section{REFERENCES}

[1] Tao Jiang and Yiyan Wu. An Overview: Peak-to-Average Power Ratio Reduction Techniques for OFDM Signals. IEEE Transactions on Broadcasting, vol. 54, no. 2, pp. 257-268, 2008.

[2] S. Katam and P. Muthuchidambaranathan. Modified SLM method for reduction of PAPR in OFDM systems using decimal sequences. 2015 IEEE International Conference on Signal Processing, Informatics, Communication and Energy Systems (SPICES), 2015.

[3] P. Weitkemper, J. Bazzi, K. Kusume, A. Benjebbour and Y. Kishiyama. Adaptive filtered OFDM with regular resource grid. 2016 IEEE International Conference on Communications Workshops (ICC), 2016.

[4] K. Hu and A. Armada. SINR analysis of OFDM and f-OFDM for machine type communications. 2016 IEEE 27th Annual International Symposium on Personal, Indoor, and Mobile Radio Communications (PIMRC), 2016.

[5] X. Zhang, M. Jia, L. Chen, J. Ma and J. Qiu. Filtered-OFDM - Enabler for Flexible Waveform in the 5th Generation Cellular Networks. 2015 IEEE Global Communications Conference (GLOBECOM), 2015.

[6] P. Guan, D. Wu, T. Tian, J. Zhou, X. Zhang, L. Gu, A. Benjebbour, M. Iwabuchi and Y. Kishiyama. 5 G Field Trials - OFDM-based Waveforms and Mixed Numerologies. IEEE Journal on Selected Areas in Communications, pp. 1-1, 2017.

[7] J. Abdoli, M. Jia and J. Ma. Filtered OFDM: A new waveform for future wireless systems. 2015 IEEE 16th International Workshop on Signal Processing Advances in Wireless Communications (SPAWC), 2015.

[8] X. Cheng, Y. He, B. Ge and C. He. A Filtered OFDM Using FIR Filter Based on Window Function Method. 2016 IEEE 83rd Vehicular Technology Conference (VTC Spring), 2016.

[9] C. Rajasekhar, D. Srinivasa rao, V. Yaswanth Raghava and D. Hanith. PAPR reduction performance in OFDM systems using channel coding techniques. 2014 International Conference on Electronics and Communication Systems (ICECS), 2014.

[10] G. Toor, H. Singh and A. Bhandari. PAPR reduction and BER improvement by using logrithmic companding hybrid with SLM technique in bit interleved COFDM system. 2014 5th International Conference - Confluence The Next Generation Information Technology Summit (Confluence), 2014.

[11] A. Eltholth. Probability distribution equalization scheme for PAPR reduction in NC-OFDM systems. 10th International Conference on Wireless Communications, Networking and Mobile Computing (WiCOM 2014), 2014.

[12] F. Juwono and D. Gunawan. PAPR reduction using Huffman coding combined with clipping and filtering for OFDM transmitter. 2009 Innovative Technologies in Intelligent Systems and Industrial Applications, 2009.

[13] Jian Zhao. Improved Performance of Optical F-OFDM over Conventional OFDM for Residual Frequency Offset Compensation. 39th European Conference and Exhibition on Optical Communication (ECOC 2013), 2013.

[14] S. Wang, J. Thompson and P. Grant. Closed-Form Expressions for ICI/ISI in Filtered OFDM Systems for Asynchronous 5G Uplink. IEEE Transactions on Communications, pp. 1-1, 2017.

[15] Jialing Li, K. Kearney, E. Bala and Rui Yang. A resource block based filtered OFDM scheme and performance comparison. ICT 2013, 2013.

[16] Xin Yu, Yu Guanghui, Yan Xiao, Yang Zhen, Xu Jun and Gao Bo. FB-OFDM: A novel multicarrier scheme for 5G. 2016 European Conference on Networks and Communications (EuCNC), 2016.

[17] M. Kasaiah and K. Babulu. Comparative performance analysis of FIR and IIR filters based on error correction codes. 2016 International Conference on Communication and Electronics Systems (ICCES), 2016.

[18] H. Rakshit and M. Ullah. A comparative study on window functions for designing efficient FIR filter. 20149 th International Forum on Strategic Technology (IFOST), 2014.

[19] Y. Fang, V. Stankovic, S. Cheng and E. Yang. Analysis on Tailed Distributed Arithmetic Codes for Uniform Binary Sources. IEEE Transactions on Communications, pp. 1-1, 2016.

[20] P. Liu, J. Chen and Y. Lin. A Hardwired Context-Based Adaptive Binary Arithmetic Encoder for H. 264 Advanced Video Coding. 2007 International Symposium on VLSI Design, Automation and Test (VLSI-DAT), 2007.

[21] N. Kumar, W. Xiang and Y. Wang. An FPGA-based fast two-symbol processing architecture for JPEG 2000 arithmetic coding. 2010 IEEE International Conference on Acoustics, Speech and Signal Processing, 2010.

[22] W. Wang and W. Zhang. Adaptive Spatial Modulation Using Huffman Coding. 2016 IEEE Global Communications Conference (GLOBECOM), 2016.

[23] . Sudha, B. Anilkumar, M. Samatha and D. Kumar. A low-complexity modified SLM with new phase sequences for PAPR reduction in OFDM system. 2015 Annual IEEE India Conference (INDICON), 2015.

[24] B. Gupta and D. Saini. BER Analysis of Space-Frequency Block Coded MIMO-OFDM Systems Using Different Equalizers in Quasi-Static Mobile Radio Channel. 2011 International Conference on Communication Systems and Network Technologies, 2011.

[25] H. Myung and D. Goodman, Single Carrier FDMA: A New Air Interface for Long Term Evolution, 1st ed. John Wiley \& Sons, 2008. 